TITLE:

\title{
Mechanism of strain-induced crystallization in filled and unfilled natural rubber vulcanizates
}

\section{$\operatorname{AUTHOR}(S)$ :}

Poompradub, S; Tosaka, M; Kohjiya, S; Ikeda, Y; Toki, S; Sics, I; Hsiao, BS

\section{CITATION:}

Poompradub, S ... [et al]. Mechanism of strain-induced crystallization in filled and unfilled natural rubber vulcanizates. JOURNAL OF APPLIED PHYSICS 2005, 97(10): 103529.

\section{ISSUE DATE:}

2005-05-15

URL:

http://hdl.handle.net/2433/50372

\section{RIGHT:}

Copyright 2005 American Institute of Physics. This article may be downloaded for personal use only. Any other use requires prior permission of the author and the American Institute of Physics. 


\title{
Mechanism of strain-induced crystallization in filled and unfilled natural rubber vulcanizates
}

\author{
Sirilux Poompradub, Masatoshi Tosaka, a) and Shinzo Kohjiya \\ Institute for Chemical Research, Kyoto University, Uji, Kyoto 611-0011, Japan \\ Yuko lkeda \\ Kyoto Institute of Technology, Matsugasaki, Kyoto 606-8585, Japan
}

Shigeyuki Toki, Igors Sics, and Benjamin S. Hsiao

Department of Chemistry, State University of New York at Stony Brook, New York 11794-3400

(Received 5 November 2004; accepted 11 March 2005; published online 13 May 2005)

\begin{abstract}
Structure evolution during deformation of unfilled natural rubber (NR) vulcanizate and filled ones with carbon black or calcium carbonate was investigated by the synchrotron x-ray diffraction. The crystallization onset strain, $\alpha^{0}$, was found to decrease by the inclusion of the filler. However, corrected $\alpha^{0}$ values into the effective strain ratio of deformable rubber portion were almost constant between filled and unfilled samples. Accordingly, our model of strain-induced crystallization of unfilled NR vulcanizates, assuming that melting temperature is independent of network-chain length $(n)$, was applied to the filled samples. The discrepancy between classical theories and experimental results was thought to come from the distribution of $n$. By the inclusion of filler, the lateral crystallite size was decreased but the orientational fluctuation increased. The lattice of the strain-induced crystallites changed almost linearly with the nominal stress. In addition, the degree of lattice deformation decreased with the filler content, especially in the carbon black-filled system. All these experimental results are consistent with the proposed model. (C) 2005 American Institute of Physics. [DOI: 10.1063/1.1900927]
\end{abstract}

\section{INTRODUCTION}

An elastomer is a polymer material which has the ability to resume its shape after large deformation. The long polymer chains of an elastomer are cross-linked with each other and account for the flexible nature of the material. Among various types of elastomers, natural rubber (NR) is the most typical one and an indispensable material for many industrial and household applications. ${ }^{1}$ The versatility of NR is mainly due to its outstanding tensile properties and the good crack growth resistance, which originates in its ability to crystallize upon elongation. Thus the strain-induced crystallization in cross-linked polymer system is a significant subject from both academic and industrial viewpoints. Even though the strain-induced crystallization of NR was recognized long ago, ${ }^{2,3}$ the crystallization mechanism in cross-linked NR had not been much understood in spite of the extensive studies on this subject. ${ }^{2-17}$

There is a characteristic feature in the wide-angle $\mathrm{x}$-ray diffraction (WAXD) patterns of highly strained NR, which is the coexistence of highly oriented crystalline reflections and near isotropic amorphous halo. This feature has been described in the early work by Katz in $1925 .^{2}$ Mitchell reported the same feature almost six decades later, and investigated the crystallinity and the degree of molecular orientation using the conventional WAXD technique. ${ }^{13}$ One may understand the peculiarity of this feature when the WAXD patterns of other semicrystalline linear polymers, e.g., poly(ethylene

\footnotetext{
${ }^{\text {a) }}$ Author to whom correspondence should be addressed; FAX: +81-774-383067; electronic mail: tosaka@ @scl.kyoto-u.ac.jp
}

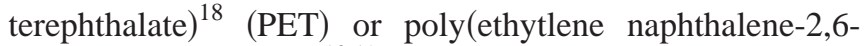
dicarboxylate) (PEN) ${ }^{18,19}$ during the uniaxial deformation were compared with the case of NR. In the case of PET or PEN, as the strain ratio increases, the amorphous halo is localized onto the equator and subsequently crystalline reflections appear, indicating that the overall molecular orientation occurs first and the crystallization follows. Despite the peculiarity, because the isotropic halo from the strained NR could be expected as a result of relaxation during long exposure using a conventional $x$-ray source, not much attention has been paid. In the subsequent synchrotron WAXD studies, this feature of NR was more clearly recognized by timeresolved WAXD measurements. ${ }^{14,20,21}$ Since synchrotron $\mathrm{x}$ ray allowed the in situ examination of structural evolution during deformation, the detection of a persistent isotropic amorphous halo in the highly strained NR cannot be related to the relaxation of the stretched chains but to an intrinsic feature of the NR samples.

In the recent quantitative studies by some of the authors of the present paper, the feature was attributed to the coexistence of stretched chains and random coiled chains. ${ }^{20-22} \mathrm{~A}$ model mechanism has been presented for the strain-induced crystallization of unfilled NR on the basis of the features found through a close inspection of the WAXD patterns, considering the polydisperse distribution of the network-chain length which results in the inhomogeneous deformation of the molecular network of NR vulcanizates. ${ }^{22,23}$ This model will be extended to the filled system.

In this paper, we discuss the structure evolution and its mechanical response in NR samples containing nanosized particulate fillers. Since the filled NR (e.g., with carbon 
TABLE I. Formulations of the NR compounds in parts by weight per hundred parts of rubbers.

\begin{tabular}{|c|c|c|c|c|c|}
\hline Ingredients & NR-0 & $\mathrm{NR}-20 \mathrm{H}$ & $\mathrm{NR}-40 \mathrm{H}$ & NR-40CA & NR-60CA \\
\hline NR & 100 & 100 & 100 & 100 & 100 \\
\hline Stearic acid & 2.0 & 2.0 & 2.0 & 2.0 & 2.0 \\
\hline Active $\mathrm{ZnO}$ & 1.0 & 1.0 & 1.0 & 1.0 & 1.0 \\
\hline $\mathrm{CBS}^{\mathrm{a}}$ & 1.0 & 1.0 & 1.0 & 1.0 & 1.0 \\
\hline Sulfur & 1.5 & 1.5 & 1.5 & 1.5 & 1.5 \\
\hline Carbon black & $\cdots$ & 20 & 40 & $\cdots$ & $\cdots$ \\
\hline Calcium carbonate & $\cdots$ & $\cdots$ & $\cdots$ & 40 & 60 \\
\hline Curing time $^{\mathrm{b}}(\mathrm{min})$ & 14 & 14 & 14 & 15 & 15 \\
\hline Volume fraction of filler, $\phi$ & 0 & 0.097 & 0.177 & 0.135 & 0.190 \\
\hline
\end{tabular}

${ }^{\mathrm{a}} N$-cyclohexyl-2-benzothiazole sulfenamide.

${ }^{\mathrm{b}}$ Curing temperature $140{ }^{\circ} \mathrm{C}$.

black) is of extreme importance in many applications, ${ }^{24}$ clarification of the relationship between the filler and the strain-induced crystallites is also an important subject from both academic and industrial viewpoints. We have reported a short communication obtained by the synchrotron WAXD and simultaneous stress-strain measurements for the carbon black-filled samples. ${ }^{25}$ Hereafter, we present additional and comprehensive results including those for the samples mixed with calcium carbonate, and discuss the strain-induced crystallization and mechanical response of the filled samples in view of the model for strain-induced crystallization described in the previous papers based on unfilled samples. ${ }^{22,23}$ An important problem on the application of the classical theories ${ }^{4,26}$ to the real system is also addressed in this paper.

\section{EXPERIMENT}

The grade of NR used in this study was ribbed smoked sheet (RSS) No. 1 from Malaysia. Carbon black (HS-HAF, Diablack N339) with an average particle size of 26-30 nm and ultrafine calcium carbonate $\left(\mathrm{CaCO}_{3}, \mathrm{ACTIFORD700)}\right.$ with an average particle size of $20 \mathrm{~nm}$ were used as fillers. The formulations for the preparation of varying rubber compounds are shown in Table I, along with the volume fraction, $\phi$, of the filler. Each of the filler was dried in an oven at $120^{\circ} \mathrm{C}$ for $2 \mathrm{~h}$. Subsequently, all of the ingredients were mixed on a two-roll mill at room temperature. The cure time was determined on the basis of cure characteristics from a JSR Curelastmeter III oscillating disk rheometer, which was used to monitor the progress of curing. The NR compounds were vulcanized into sheets of 1-mm thickness under pressure in a mold. Dumbbell-shaped specimens were cut from the sheets. The initial thickness and the width of the thin part of the specimen were 1 and $3 \mathrm{~mm}$, respectively.

Synchrotron WAXD experiments and simultaneous tensile measurements were carried out at the $\mathrm{X} 3 \mathrm{~A} 2$ beamline in national synchrotron light source (NSLS), Brookhaven National Laboratory (BNL), NY, USA. The wavelength of the $x$ ray was $0.1542 \mathrm{~nm}$. The two-dimensional (2D) WAXD patterns were recorded in every $30 \mathrm{~s}$ by a charge-coupled device (marCCD x-ray detector system) with a flat screen and a pixel size of $0.158 \mathrm{~mm}$. The camera length was $93.5 \mathrm{~mm}$ (for NR-0 and NR-40H) or $107.0 \mathrm{~mm}$ (for NR-20H, NR-40CA, and NR-60CA). These experimental conditions enabled us to detect the change of diffraction angle which corresponds to variation of lattice spacing smaller than $0.04 \mathrm{~nm}$.

A tabletop stretching machine from Instron, Inc., modified to allow the symmetric deformation of the sample, was used to illuminate the same sample position during stretching with the focused $\mathrm{x}$ ray. ${ }^{21,25,27-30}$ The incident beam impinged on the sample perpendicular to the stretching direction. Each specimen was continuously elongated up to the predetermined strain ratio and then retracted to the original length, not stopping during the exposure. The strain ratio, $\alpha$, was determined from the distance between the clamps during the deformation. Here, $\alpha=l / l_{0}$ in which $l_{0}$ is the initial length of the sample and $l$ is that of the elongated one. The initial distance between the clamps was $25 \mathrm{~mm}$ and the deformation rate was $10 \mathrm{~mm} / \mathrm{min}$. All the measurements were carried out at room temperature $\left(\mathrm{ca} .25^{\circ} \mathrm{C}\right)$.

\section{RESULTS}

\section{A. Tensile properties}

The results of the tensile stress-strain measurements are shown in Fig. 1. For the filled samples, the stress upturn occurred at a smaller $\alpha$ value, compared to the unfilled sample. This effect was more significant for the samples with carbon black than those with calcium carbonate, according to the comparison of, e.g., NR-40H and NR-60CA having closer $\phi$ values. This is in accord with the general trend reported previously. ${ }^{31}$ Although calcium carbonate of nanometer size (ACTIFORD700) was used, its effect on the tensile behaviors of NR was less than that of a conventional carbon black [high abration furnace (HAF)].

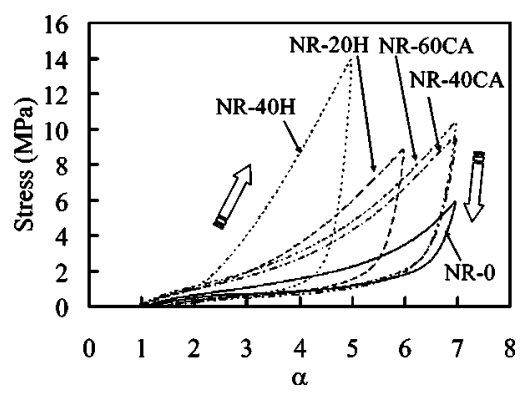

FIG. 1. Stress-strain curves. 

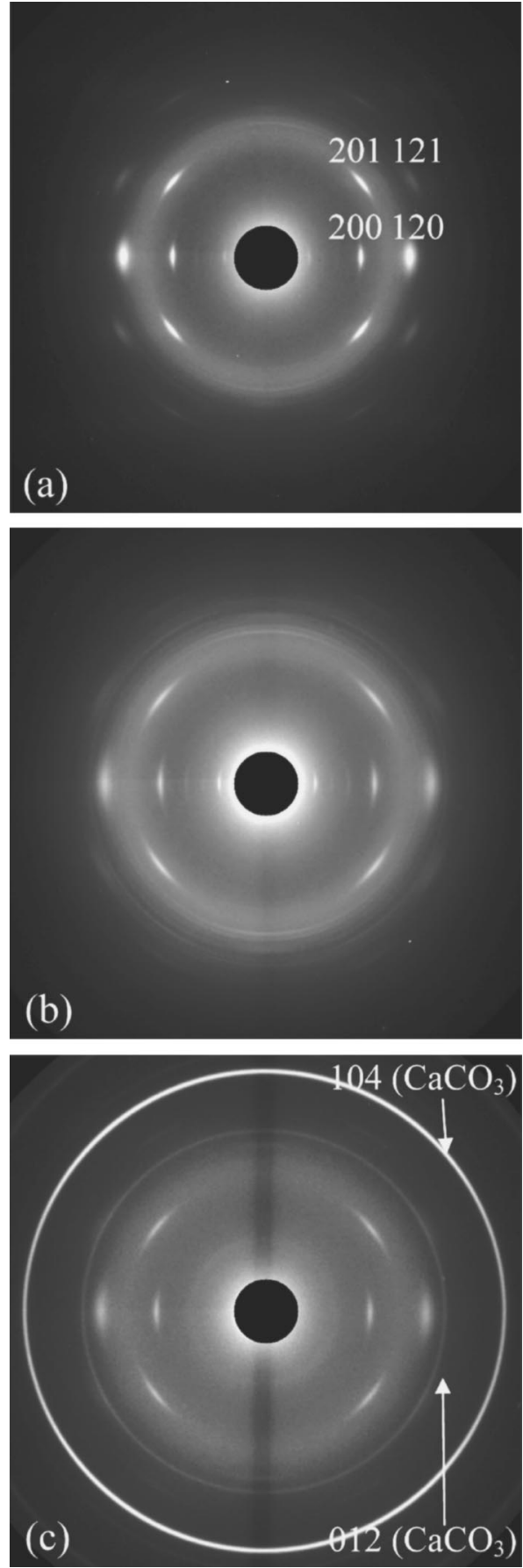

FIG. 2. Flat camera WAXD patterns of (a) NR-0, (b) NR-20H, and (c) NR-40CA, at strain ratio $\alpha=5$. Stretching direction is vertical.

\section{B. WAXD patterns of unfilled and filled rubber samples}

Figure 2 shows the examples of the WAXD patterns at $\alpha=5$. Only the highly oriented crystal reflections in WAXD are observed in NR. On the other hand, the ring-shaped (unoriented) reflections of calcium carbonate (calcite), which can be indexed as 012 (weak inner one) and 104 (strong outer one) reflections of the trigonal crystal (a $=0.4989 \mathrm{~nm}, c=1.7062 \mathrm{~nm}$, space group No. $167: R \overline{3} c),{ }^{32}$ are seen in the WAXD pattern of NR-40CA. As confirmed by the ring-shaped reflections, the filler particles did not have a preferred orientation in the rubber samples upon elongation.
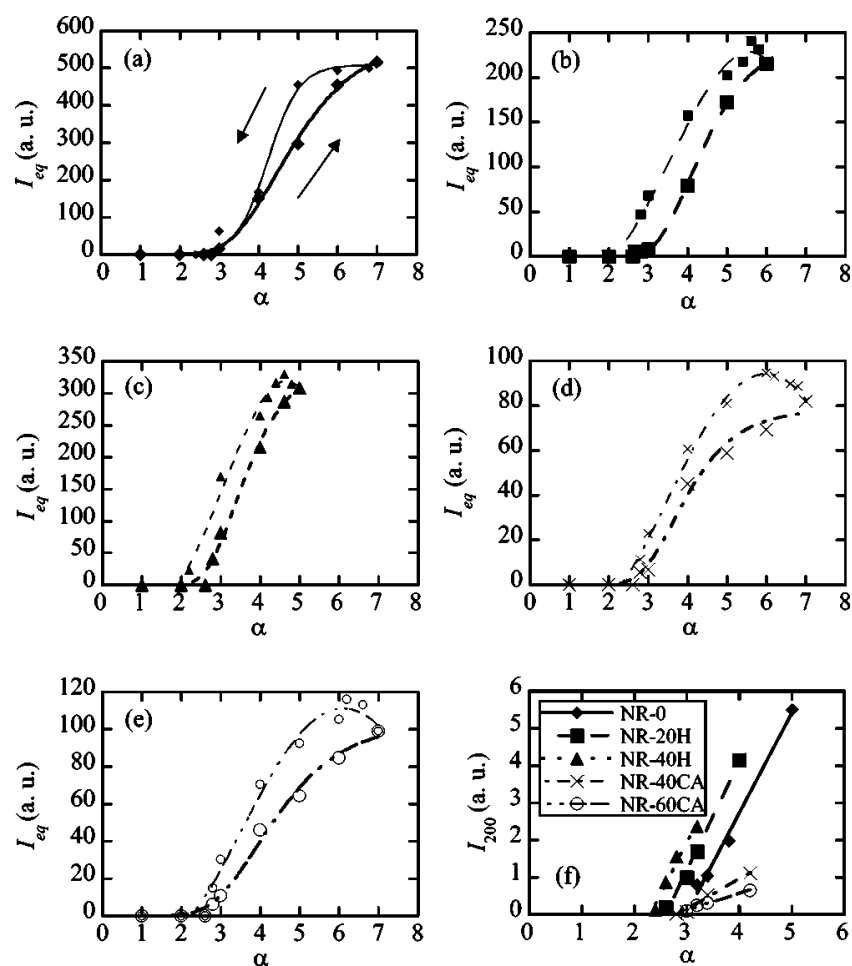

FIG. 3. Variation of the integrated intensity of the equatorial reflections, $I_{\mathrm{eq}}$, during the stretching and retracting processes for (a) NR-0, (b) NR-20H, (c) NR-40H, (d) NR-40CA, and (e) NR-60CA. The thick and thin lines indicate the stretching and the retracting processes, respectively. The larger symbols correspond to the stretching process. The $I_{200}$ data during the stretching process near the onset of crystallization are shown in part (f), from which the $\alpha^{0}$ values were estimated.

If some sites on the filler particle were acting as nucleating points, randomly oriented crystallites of NR would have appeared, resulting in ring-shaped reflections of NR crystallites. Such ring-shaped reflections of NR have not been found in this study or in the previous studies. ${ }^{22,23}$ The absence of such randomly oriented reflection of NR crystallites strongly suggests that both carbon black and calcium carbonate in this study are not acting by themselves as nucleating agents.

\section{Development of strain-induced crystallites}

The development of crystallinity was evaluated by using the integrated intensity of 200 and 120 reflections $\left(I_{\mathrm{eq}}\right)$, obtained by a conventional curve-fitting method, from the equatorial intensity distribution that included $\pm 25^{\circ}$ from the equator. Figures 3(a)-3(e) show the development of $I_{\text {eq }}$ with $\alpha$. During the stretching process, the $I_{\mathrm{eq}}$ value of all the samples increased monotonically. In addition, during the retracting process, in accord with our previous studies, ${ }^{22,23}$ the $I_{\mathrm{eq}}$ value of the unfilled sample decreased monotonically [Fig. 3(a)]. In the case of the filled samples, the $I_{\text {eq }}$ value increased when the retracting process started, and then decreased monotonically [Figs. 3(b)-3(e)]. This increase would not be due to the increase of the sample thickness (i.e., the scattering mass, synchronizing the contraction of the sample) because the change in the sample thickness was not so large when the sample was highly stretched, and furthermore, such an increase in intensity has not been observed for unfilled 
TABLE II. Onset strain ratio of crystallization for unfilled and filled NR samples.

\begin{tabular}{lcccc}
\hline \hline & \multicolumn{3}{c}{ Onset strain ratio of crystallization } \\
Sample code & $V^{\mathrm{a}}$ & $\alpha^{0}$ & $\alpha_{r}^{0}(\phi)$ & $\alpha_{r}^{0}(V)$ \\
\hline NR-0 & $\ldots$ & 3.0 & 3.0 & $\ldots$ \\
NR-20H & 0.167 & 2.6 & 2.8 & 2.9 \\
NR-40H & 0.305 & 2.3 & 2.6 & 2.9 \\
NR-40CA & $\ldots$ & 2.8 & 3.1 & $\ldots$ \\
NR-60CA & $\ldots$ & 2.7 & 3.1 & $\ldots$ \\
\hline
\end{tabular}

${ }^{\mathrm{a}} V / \phi=1.72$

${ }^{\mathrm{b}} \alpha^{0}$ : the values before correction, $\alpha_{r}^{0}(\phi)$ : corrected values for the filler volume fraction, and $\alpha_{r}^{0}(V)$ : corrected values for the volume fraction of the fillers plus occluded rubber.

samples. ${ }^{22,23}$ Therefore, the increase in $I_{\mathrm{eq}}$ at the beginning of the retracting process was mainly attributed to the increase in the crystallinity of the sample.

In this study, the strain ratio at the onset of crystallization $\left(\alpha^{0}\right)$ was determined by the relation between diffraction intensities and $\alpha: \alpha^{0}$ value was estimated as the intercept of the regression line in the plot of $I_{200}$ against $\alpha$ near the crystallization onset region. (Here, $I_{200}$ denotes the integrated intensity of the 200 reflection of NR.) In this case, the 120 reflection was excluded from the evaluation of diffraction intensity because the 120 reflection is overlapped by the strong amorphous halo, and hence separation of the weak peak from the halo was impossible when crystallinity was low. The plots used for the estimation of the $\alpha^{0}$ values are shown in Fig. 3(f). Table II shows the $\alpha^{0}$ values determined in this way. It was found that $\alpha^{0}$ shifted to a lower value in the case of the samples mixed with filler. This trend is more pronounced for the samples filled with carbon black than those with calcium carbonate.

\section{Crystallite size}

The crystallite size was estimated by using the Scherrer equation,

$$
L_{200}=K \cdot \lambda /(\beta \cos \theta),
$$

where $L_{200}$ is the crystallite size in the direction perpendicular to the (200) plane, $\lambda$ is the wavelength, $\beta$ is the halfwidth of the 200 reflection in the radial direction, $\theta$ is the Bragg angle (half of the scattering angle), $K$ is a constant, and the value of 0.89 was used in this study. ${ }^{33}$ The detailed procedure for the estimation of $L_{200}$ has been described in our previous paper. ${ }^{22}$ Figure 4 shows the variation of $L_{200}$ during the stretching and retracting processes. As shown in the previous paper, ${ }^{22}$ the $L_{200}$ values of all the samples decreased during the stretching process, whereas the values increased gradually during the retracting process. On the whole, the $L_{200}$ values of the filled samples were smaller than those of the unfilled one.

\section{E. Orientational fluctuation of crystallites}

The degree of crystallite orientation was evaluated based on the azimuthal intensity distribution of the 200 reflection. The azimuthal half-width, $\beta_{\mathrm{az}}$, of the intensity distribution was estimated as described in our previous paper. ${ }^{22}$ The
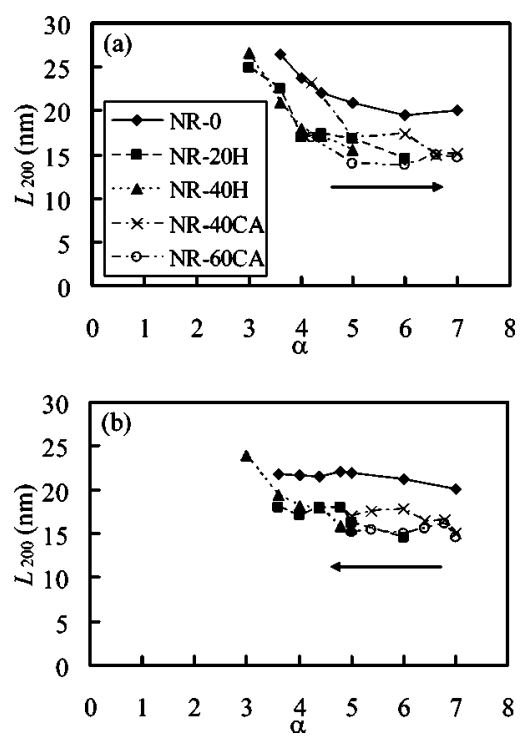

FIG. 4. Variation of lateral crystallite size, $L_{200}$, during the (a) stretching and (b) retracting processes.

smaller value of $\beta_{\mathrm{az}}$ indicates the smaller fluctuations in orientation. Figure 5 shows the variation of $\beta_{\mathrm{az}}$ during the stretching and the retracting processes. For each sample, the orientational fluctuation decreased with $\alpha$ during stretching, and then increased gradually during retracting. It was noted that the $\beta_{\mathrm{az}}$ values of all the filled samples were larger than the unfilled ones.

\section{F. Lattice deformation of strain-induced crystallites}

In order to evaluate the deformation of the crystal lattice by the stress upon stretching and retracting processes, the lattice constants were estimated from the WAXD patterns during deformation by using the least-square regression method. The unit cell was assumed to be a rectangular parallelepiped, according to the structure analysis by Nyburg. ${ }^{34}$ The estimated values of the lattice constants and the volume
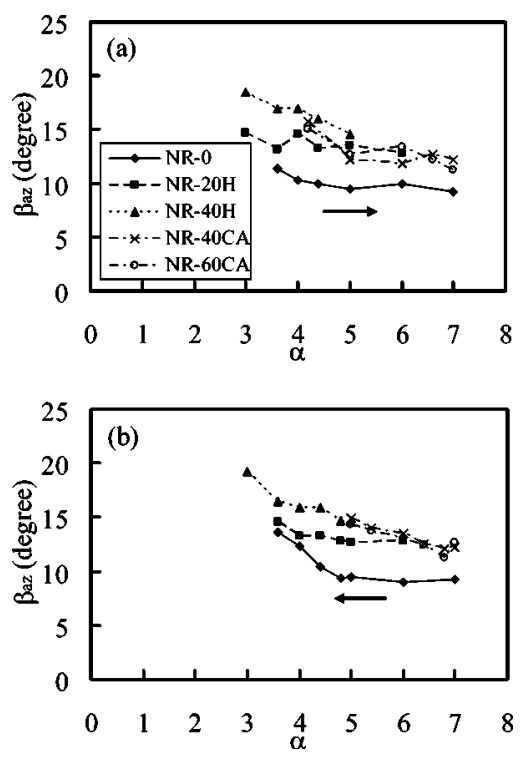

FIG. 5. Variation of azimuthal half-width of 200 reflection, $\beta_{\mathrm{az}}$, during the (a) stretching and (b) retracting processes. 

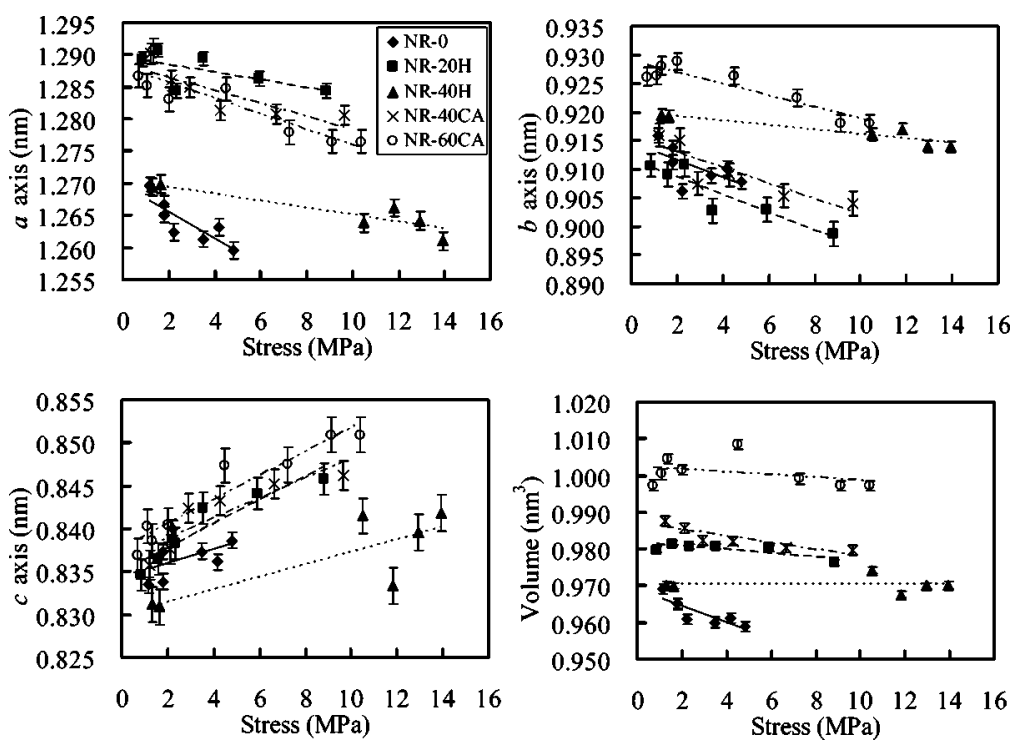

FIG. 6. Lattice constants and volume of the unit cell as a function of nominal stress. Both the stretching and retracting processes are included. of the unit cell are plotted in Fig. 6 against the stress values obtained in the dynamic experiment. We note that both the stretching and retracting processes are included in this figure. The contraction of the unit cell in the $a$ and $b$ directions (perpendicular to the stretching direction), and the elongation along the $c$ direction (parallel to the stretching direction) with the increase in the nominal stress were recognized in both the unfilled and filled samples. The differences of the lattice constants among the samples at zero stress, which were estimated by the extrapolation of the plots, may be due to the slight differences of the experimental configurations and corresponding errors. Unfortunately, measurements for NR-0 and NR-40H were performed at different experimental periods. The differences of the absolute values of the lattice constants are, therefore, not discussed in this paper.

\section{DISCUSSIONS}

\section{A. The model of strain-induced crystallization for unfilled NR}

In our previous papers, the following model has been presented for the strain-induced crystallization of unfilled NR. ${ }^{22,23}$

When the stretching of a NR vulcanizate is started, the network chains in the sample are divided into highly stretched chains and coiled chains, as a result of inhomogeneous deformation due to inhomogeneous topology of chains. (In the field of polymer physics, the term "coiled" is customary used to express the tangled chain conformation in general. ${ }^{35}$ ) The coiled chains result in the nearly isotropic amorphous halo in the WAXD pattern. ${ }^{20-23,30}$ Upon further elongation, the melting temperature $\left(T_{m}\right)$ of the sample is increased due to elongation of the coiled chains. ${ }^{3,4,7,8,11,16,22,23}$ When $T_{m}$ exceeds the ambient temperature, the system enters a supercooled state because polymer molecules cannot crystallize otherwise under sufficient supercooling. ${ }^{36}$ Subsequently, the oriented crystallites will be formed onto the stretched chains. The molecular chains that are consumed for the crystal growth (in the same way as the shishi-kebab structure ${ }^{37-41}$ ) would be supplied by the surrounding coiled chains.

As a result of crystallization, the molecular chains are partly extended in the stretching direction, and the rest, namely, the amorphous portion, is conversely relaxed. Thus the entropy of the amorphous portion will be increased after crystallization, ${ }^{4,15,22,30,42}$ and as a result, further crystallization would be hindered without additional elongation of the sample. The persistence of almost isotropic amorphous halo in the WAXD pattern of highly strained NR samples ${ }^{20,21,30}$ can be explained in this way.

An important postulate assumed in the previous studies is that $T_{m}$ of the strain-induced crystallites is almost independent of network-chain density, $\nu$. This postulate arises from the result that the $\alpha^{0}$ values for unfilled NR vulcanizates were almost independent of $\nu^{22,23}$ If $T_{m}$ were a function of both $\alpha$ and $\nu, \alpha^{0}$ (where $T_{m}$ minus supercooling equals to ambient temperature) would depend on $\nu$. However, not only in our previous studies ${ }^{22,23}$ but also in another synchrotron study, ${ }^{43} \alpha^{0}$ was found to be almost independent of $\nu$. Even when the temperature was changed by the ambient condition, $\alpha^{0}$ was still independent of $\nu$. The latter result was implicitly reported on cross-linked $\mathrm{NR}{ }^{7}$ by plotting the data of $\alpha^{0}$ against crystallization temperature (in Table IV of Ref. 7), we can obtain curves for three samples with different $\nu$, which are almost superposed with each other as shown in Fig. 7. At least, in the case of "temperature-induced" crystallites, $T_{m}$ is independent of $\nu$ according to the dilatometric study $^{44}$ of a series of NR samples with different $\nu$ values, though the growth rate of the temperature-induced crystals ${ }^{42}$ was suppressed by increasing $\nu{ }^{5}$ In this way, this postulate is based on sufficient experimental evidences as stated above.

On the other hand, classical theories of strain-induced crystallization for homogeneous network have predicted that $T_{m}$ depends on the network-chain length, that is, on $\nu^{4,11,45}$ However, there is not enough evidence to prove that $T_{m}$ depends on $\nu$ for the strain-induced crystallites of vulcanized NR. For example, in Ref. 7, the results shown in Fig. 7 were analyzed by using the Flory's equation, ${ }^{4}$ 


$$
\frac{1}{T_{i}}=\frac{1}{T_{i}^{0}}-\frac{R}{\Delta H} \psi(\alpha, n)
$$

where

$$
\psi(\alpha, n)=\left(\frac{6}{\pi n}\right)^{1 / 2} \alpha-\frac{\left(\frac{\alpha^{2}}{2}+\frac{1}{\alpha}\right)}{n},
$$

with $T_{i}$ being the incipient crystallization temperature (namely, the ambient temperature where crystallization starts at strain ratio $\alpha), T_{i}^{0}$ a constant, $R$ the gas constant, $\Delta H$ the melting enthalpy, and $n$ the number of statistical units per network chain. The value $n$ is not equal to the number of repeating unit per chain, $N$. The ratio $N / n$ is a measure of rigidity of the chain. The data that give the plots in Fig. 7 for the samples with different $N$ have led to different values of $\Delta H$ and $N / n$ for each sample. In this way, the dependence of $T_{m}$ on $\nu$ has been believed unquestioningly by regarding other constants as fitting parameters. However, it is unreasonable to attribute different values of $\Delta H$ and $N / n$ for the series of NR samples prepared in the similar manner.

The discrepancy between the experimental results and the theoretical prediction may arise from the inhomogeneous nature of the chemically cross-linked NR networks. That is, the premise of the classical theories for the homogeneous network may not be valid in the real NR network system. The coexistence of highly oriented crystalline reflections and nearly isotropic amorphous halo in the WAXD pattern (Fig. 2) suggests the coexistence of highly stretched network chains and coiled chains, as stated before. ${ }^{20-22}$ This would be due to a polydisperse but finite distribution of network-chain length in the real system. A theoretical treatment for such realistic systems, considering the distribution of networkchain length, has been presented by Dietrich et al. ${ }^{46}$ Though their model for strain-induced crystallization was different from ours and the theory failed to predict the experimental trends of the crystallization onset strain, the coexistence of highly stretched chains and coiled ones was successfully predicted in their paper.

The hypothesis of the coexistence of two types of network chains has led us to the above-discussed model to satisfy all the experimental evidences consistently. Hereafter, we discuss the results for filled samples from this viewpoint.

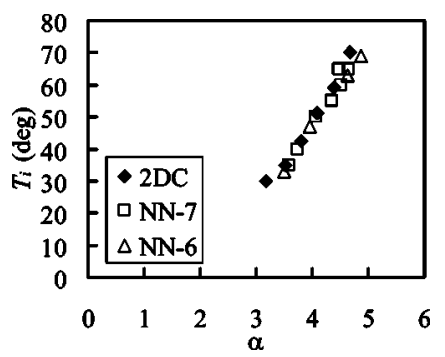

FIG. 7. Relationship between incipient crystallization temperature, $T_{i}$, and $\alpha$. The data in Ref. 7 were used for the plot. The number of repeating unit per chain, $N$, for samples 2DC, NN-7, and NN-6 are 198, 140, and 154, respectively. 2DC was cured with dycumil peroxide, and NN-7 and NN-6 were cross-linked by irradiating with $\gamma$ radiation.

\section{B. Onset of crystallization}

As shown in Table II, the strain-induced crystallization of the filled samples starts at a smaller strain ratio than the unfilled sample. This shift of $\alpha^{0}$ by the inclusion of carbon black filler has already been reported by Gehman and Field. ${ }^{47}$ To explain these results, we have to take account that the filler particles do not deform and only the rubber portion can be deformed. Because of the existence of the filler particles, the effective strain ratio of the rubber portion is larger than the nominal (macroscopic) one. In the simplest model, where no deformation of filler particles and uniform deformation of the rubber portion are assumed, the relationship between the filler volume fraction $\phi$ and the effective strain ratio $\alpha_{r}(\phi)$ can be formulated as ${ }^{48}$

$$
\alpha_{r}(\phi)=(\alpha-\phi) /(1-\phi) .
$$

Of course, the real value of the effective strain ratio depends on the network structure and the aggregation of filler particles, but we tentatively adopt this equation as the starting point. The $\alpha^{0}$ values are converted using Eq. (3) and the values, $\alpha_{r}^{0}(\phi)$, are shown in Table II. The $\alpha_{r}^{0}(\phi)$ values are closer to each other compared to the $\alpha^{0}$ values.

Two types of correction for the usage of Eq. (3) are considered to explain the relatively large deviation of $\alpha_{r}^{0}(\phi)$ for samples with carbon black (NR-20H and NR-40H) than those with calcium carbonate (NR-40CA and NR-60CA). In the case of carbon black-filled NR, formation of "bound rubber" layer which is confined (both physically and chemically) around the filler particle is widely known, ${ }^{49-51}$ and the tendency of calcium carbonate is known to be less enhanced. Equation (3) assumes that the whole portion of rubber deforms uniformly while the filler particles do not deform. If we further assume that the bound rubber layer does not deform upon elongation as well as the filler particles, the smaller $\alpha_{r}^{0}(\phi)$ values in Table II for the carbon black-filled samples may be explained by the underestimation of the volume fraction of undeformable components. In the reverse way, we calculated the thickness of the bound rubber layer from $\alpha^{0}$ to be ca. $3-4 \mathrm{~nm}$. (The calculation procedure is described in the Appendix.) The calculated thickness of the bound rubber is within the range of reported values. ${ }^{50,52}$

The other type of correction considers the aggregate structure formed by the elementary particles of carbon black. ${ }^{53}$ The aggregate of carbon black particles traps a part of matrix rubber in the internal void space, and accordingly, the effective volume of the aggregate is larger than the net volume of carbon black particles. The trapped rubber in the carbon black aggregate is called "occluded rubber" and also contributes to the undeformable component. The effective volume fraction, $V$, of carbon black including the occluded rubber can be estimated by using dibutylphthalate absorption (DBPA) values in $\mathrm{cm}^{3} / 100 \mathrm{~g}$ as 54

$$
V / \phi=0.5\{1+[1+0.02139(\mathrm{DBPA})] / 1.46\} .
$$

In the case of the carbon black used in this study (HSHAF N339), DBPA is about $120 \mathrm{~cm}^{3} / 100 \mathrm{~g}$ (according to ASTM D-1765) $)^{53}$ and the corresponding value of $V / \phi$ is calculated to be 1.72 by using Eq. (4). The corrected $\alpha^{0}$ 
based on Eq. (3) by using $V$ instead of $\phi\left[\right.$ namely, $\left.\alpha_{r}^{0}(V)\right]$ for NR-20H and NR-40H are shown in Table II along with the values of $V$. The deviations of $\alpha_{r}^{0}(V)$ values for the carbon black-filled samples from the $\alpha^{0}$ value of the unfilled sample are comparable with those of $\alpha_{r}^{0}(\phi)$ for the calcium carbonate-filled samples. Slight fluctuations of these values may be partly due to the oversimplification of the model assumed for the derivation of Eq. (3).

The bound rubber and occluded rubber coexist in the real system, i.e., the undeformable rubber component would be composed of both of them. However, their relative contributions to the real effective strain are presently very difficult to estimate. Therefore, we have estimated each of the effects separately, as described above. The more detailed quantitative analysis is in the scope of future works.

Because $\alpha^{0}$ for the unfilled sample, $\alpha_{r}^{0}(\phi)$ for the calcium carbonate-filled samples, and $\alpha_{r}^{0}(V)$ for the carbon black-filled ones distribute in the small range, we may be able to assume them to be constant in the range of experimental error for the samples used in this study. Under this assumption, the model for the onset of strain-induced crystallization in our previous papers ${ }^{22,23}$ may be applicable also for the filled NR. That is, $T_{m}$ of NR would be determined as a function of the effective strain of the deformed rubber portion. Apparent increase in $\nu$ by mixing with filler would not affect $T_{m}$. Strain-induced crystallization will start when $T_{m}$ of NR is increased with the increase in the effective strain and the system enters a supercooled state. ${ }^{11,22,23}$ Here, we further assumed that the fillers do not suppress the melting temperature. This is because filler particles are too large to be incorporated in the crystal lattice of $\mathrm{NR},{ }^{34,55}$ and accordingly, they will not affect the melting temperature.

\section{Growth of crystallites}

In the previous paper, on the basis of the observations that the crystalline reflections of NR were highly oriented even when they started to appear, we proposed that the strain-induced crystals are nucleated from the stretched network chains. This assumption was consistent with the result that the values of $L_{200}$ were smaller for the samples with the larger $\nu$; the more densely crystallization starts, the smaller crystallite size results due to the limited availability of coiled network chains. Note that the resulted crystallites can be either chain-folded lamellae or sheaflike (fringed micelle) crystallites, ${ }^{35}$ depending on the longitudinal crystallite size, the available length of network chains, strain ratio, and so on. Such a morphological change with the strain ratio was suggested by Gent. ${ }^{5}$ We note that the reason for the smaller crystallite size in the samples with the larger $\nu$ should not be due to the suppressed rate of crystallization. ${ }^{44}$ Strain-induced crystallization is a fast process that has a time constant of ca. $100 \mathrm{~ms},{ }^{10}$ and accordingly, the observed size of the crystallites must reflect the size near the completion of the growth. Furthermore, suppression of crystallinity due to the higher $\nu$ has not been observed in our previous experiments. ${ }^{22,23}$

The smaller crystallite size in the filled samples (Fig. 4) can be explained in the similar way. According to transmission electron microscopy (TEM) of stretched NR samples filled with carbon black, ${ }^{56-58}$ the filler particles (or the aggregates of filler particles) are connected by dark bands running in the stretching direction. The strain-induced crystallites may be formed in the bands, considering the origin of diffraction contrast in bright-field TEM images. ${ }^{59}$ A strain analysis based on the finite element $\operatorname{method}^{60}$ (FEM) also leads to the same interpretation. The result of FEM indicates that adjacent filler particles are connected by the region of high strain in deformed rubber. Therefore, there should be much stretched chains between the filler particles. These stretched chains will lead to dense occurrence of crystallites. On the basis of the TEM and FEM studies, the smaller crystallite size of the filled samples may be attributable to the dense crystal formation on the stretched chains between the filler particles.

If the crystallites are formed from precedently stretched chains which are bundled with each other, it is very difficult to explain why the smaller crystallite size results from the more densely existing stretched chains. The reason why the stretched chains do not gather together to form crystallites must be due to their very low mobility. Being tightly stretched would be incompatible with the mobility for the network chains. In order to attain the mobility to form the crystal lattice, the network chains must be relaxed to some extent. Given this perspective, the observed increase in crystallinity [Figs. 3(b)-3(e)] at the beginning of the retracting process can be explained by the crystallization incorporating the slightly relaxed chains that attained the mobility from the stretched immobile state. The director of the stretched chains can be fluctuated to connect the filler particles. This would result in larger orientational fluctuations for the filled samples (Fig. 5).

\section{Stress field around the strain-induced crystallites}

Based on the linear relationship between the nominal stress and the lattice constants, we have proposed that the transfer of the stress to the crystallites is mediated by frames of surrounding chains. ${ }^{22}$ We can observe the similar linearity between the nominal stress and the lattice constants for the filled samples in Fig. 6. Therefore, it is conceivable that the similar mechanical model of the stress transfer for the unfilled sample can be applied to the filled ones. It is seen that the absolute values of the gradients in Fig. 6 decrease with the increase in filler content. This trend is apparent for the carbon black-filled samples, and is slightly recognized for the calcium carbonate-filled ones. The smaller degree of lattice deformation in the filled systems indicates that the smaller stress is applied to the crystallites due to the presence of filler particles. ${ }^{25}$

The mechanism on how the smaller stress is applied to the NR crystallites by the inclusion of filler particles can be explained with the schematic illustration in Fig. 8 which is drawn on the basis of the discussion in Ref. 22. (We note that there are coiled chains surrounding the frames and crystallites in Fig. 8, though the coiled chains are not drawn.) When the system is stretched in the vertical direction, the frame will compress the crystallites in the horizontal direction. If an elementary filler particle (average size: 20-30 nm) occu- 


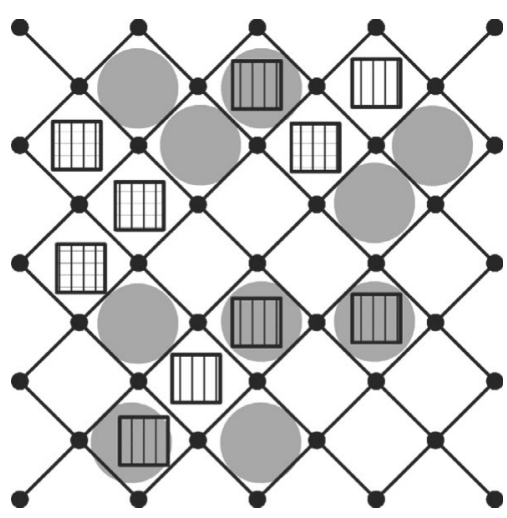

FIG. 8. Mechanical model to explain the deformation of crystallites for the filled sample. The filled squares and shaded circles represent NR crystallites and filler particles, respectively.

pies the same grid with the crystallite $\left(L_{200}: 15-30 \mathrm{~nm}\right)$, the filler particle may support some part of the compressive stress, and less stress may be apportioned to the crystallite. Though the NR crystallites and filler particles do not necessarily occupy the same grid, the average stress to the crystallites may be decreased by the inclusion of filler particles. In this way, the smaller degree of lattice deformation for the filled samples (Fig. 6) can be explained.

\section{E. Relationship among the tensile properties, crystallization, and fillers}

From the technological viewpoint, the reinforcing effect of crystallites and fillers is of much interest. There is a complicated aspect in the effect of strain-induced crystallization on the tensile properties, because crystallization may both decrease and increase the modulus depending on the situation. Stress relaxation (the decrease in modulus) due to crystallization of stretched NR vulcanizates has been reported in many studies. ${ }^{5,9,16,42}$ This is because the molecular chains partly adopt the extended conformation aligned in the stretching direction. ${ }^{4,15,22,30,42}$ On the other hand, after the consumption of crystallizable molecular chains, further extension by the crystallization is hindered and the role of crystallites as fillers to increase the modulus (namely, to harden the sample) becomes apparent. By changing the deformation rate of the rubber sample, the effect of crystallization on modulus can be recognized. The locations of stress plateau (due to relaxation) and the upturn (due to hardening) shift to the smaller strain ratio in the stress-strain curve on synthetic cis-1,4-polyisoprene rubber (IR) vulcanizates, when the deformation rate is decreased. ${ }^{42}$ It is well known that IR, the synthetic homolog of NR, is less crystalline and exhibits inferior mechanical properties, e.g., smaller values of stress at break and inferior tear strength, especially at a high deformation rate. ${ }^{61}$ The comparison of IR with NR suggests the importance of the strain-induced crystallites as filler.

The reinforcing effect of heterogeneous filler, especially of carbon black, ${ }^{48,53}$ is much straightforward compared to that of strain-induced crystals. The larger reinforcing effect of carbon black than calcium carbonate (e.g., in Fig. 1) has been explained by the formation of bound rubber layer ${ }^{49-51}$ and aggregate structure of carbon black particles. ${ }^{54}$ Though differences between carbon black and calcium carbonate were detected in the $\alpha_{r}^{0}(\phi)$ values in Table II and in the change in the slopes of the plots in Fig. 6, detailed discussion about the tensile properties, effect of filler, and crystallization requires additional experimental evidences. These points are the subjects in our future works.

\section{CONCLUSION}

The synchrotron WAXD and the simultaneous stressstrain measurements were performed for the filled and unfilled NR vulcanizates. The following results were obtained.

(1) The $\alpha^{0}$ value was shifted to the lower value by the inclusion of fillers. If we consider the effective strain ratio of the deformable rubber portion in the filled samples, the strain-induced crystallization may start at almost the same strain ratio for the unfilled sample in this study.

(2) By the inclusion of fillers, the lateral crystallite size, $L_{200}$, became smaller, while the degree of orientational fluctuation, $\beta_{\mathrm{az}}$, became larger.

(3) The lattice of the strain-induced crystallites was deformed almost linearly with the nominal stress. The degree of deformation was smaller for the samples filled with the larger amount of carbon black. The samples filled with calcium carbonate showed the similar but weaker trend.

On the basis of these results, we extended the model for the strain-induced crystallization of unfilled NR described in the previous papers, ${ }^{22,23}$ to explain the crystallization in the filled NR. The important assumption for this model is that $T_{m}$ is independent of $\nu$, which may be valid for the filled NR vulcanizates because of conclusion (1). Another assumption is that the strain-induced crystals grow consuming the surrounding coiled chains, which was supported by the observation of the small crystallite size for the filled samples.

The lattice deformation of the filled and unfilled samples could be explained by considering the pantographlike frame structure and by assuming that the crystallites tend to be compressed by the frames in the direction perpendicular to the stretching direction.

TABLE III. Values estimated for the calculation of the thickness of the bound rubber layer.

\begin{tabular}{|c|c|c|c|c|c|c|}
\hline \multirow{2}{*}{$\begin{array}{l}\text { Sample } \\
\text { code }\end{array}$} & \multirow{2}{*}{$\begin{array}{l}\text { Onset strain of } \\
\text { crystallization, } \alpha^{0}\end{array}$} & \multirow[b]{2}{*}{$\phi_{0}$} & \multirow[b]{2}{*}{$\phi_{\text {eff }}$} & \multirow[b]{2}{*}{$\left(\phi_{\mathrm{eff}} / \phi_{0}\right)^{1 / 3}$} & \multicolumn{2}{|c|}{$\begin{array}{l}\text { Thickness of the bound rubber } \\
\text { layer (nm) assuming the } \\
\text { average particle size }\end{array}$} \\
\hline & & & & & $26 \mathrm{~nm}$ & $30 \mathrm{~nm}$ \\
\hline NR-20H & 2.4 & 0.10 & 0.20 & 1.3 & 3.5 & 4.0 \\
\hline NR-40H & 2.3 & 0.18 & 0.34 & 1.3 & 3.2 & 3.7 \\
\hline
\end{tabular}


In this way, the model presented in our previous papers $^{22,23}$ could consistently explain the strain-induced crystallization of filled NR and the mechanical response of the crystallites.

\section{ACKNOWLEDGMENTS}

This work was supported partly by a Grant-in-Aid for Scientific Research (B)(2), No. 15404011, from Japan Society for the Promotion of Science, and partly by National Science Foundation (Grant No. DMR-0405432) in the USA team.

\section{APPENDIX}

The thickness of the bound rubber layer was calculated as follows. First, we assumed that the properly corrected onset strain of crystallization (namely, $\alpha_{r}^{0}$ ) was constant among the samples used in this study, i.e., $\alpha_{r}^{0}=3.0$ for all the samples [substituted to the left side of Eq. (3)]. Next, the effective volume fraction of undeformable component containing the bound rubber, $\phi_{\text {eff }}$, was calculated by solving Eq. (3) for the measured (uncorrected) $\alpha^{0}$ substituted to the right side. The $\phi_{\text {eff }}$ values calculated in this way were compared with the net volume fraction of filler, $\phi$, indicated in Table I. The fraction $\phi_{\text {eff }} / \phi$ is the volume expansion ratio of the undeformable component due to the formation of the bound rubber layer. The linear-expansion ratio of the undeformable component, namely, the expansion ratio of particle diameter, due to the formation of the bound rubber layer is $\left(\phi_{\mathrm{eff}} / \phi_{0}\right)^{1 / 3}$. The thickness of the bound rubber layer is calculated from the average particle size, $D$, of the carbon black and the linear-expansion ratio to be $D\left[\left(\phi_{\mathrm{eff}} / \phi_{0}\right)^{1 / 3}-1\right] / 2$. The values estimated for the calculation of the thickness of the bound rubber layer are indicated in Table III.

${ }^{1}$ P. W. Allen and K. P. Jones, Natural Rubber Science and Technology (Oxford University Press, Oxford, 1988), p. 1.

${ }^{2}$ J. R. Katz, Naturwiss. 19, 410 (1925).

${ }^{3}$ L. Mandelkern, Rubber Chem. Technol. 66, G61 (1993).

${ }^{4}$ P. J. Flory, J. Chem. Phys. 15, 397 (1947).

${ }^{5}$ A. N. Gent, Trans. Faraday Soc. 50, 521 (1954).

${ }^{6}$ M. H. Walters, J. Polym. Sci., Part A: Gen. Pap. 1, 3091 (1963).

${ }^{7}$ K. J. Smith, Jr., A. Greene, and A. Ciferri, Kolloid Z. Z. Polym. 194, 49 (1964).

${ }^{8}$ W. R. Krigbaum, J. V. Dawkins, G. H. Via, and Y. I. Belta, J. Polym. Sci., Part A-2 4, 475 (1966).

${ }^{9}$ H.-G. Kim and L. Mandelkern, J. Polym. Sci., Part A-2 6, 181 (1968).

${ }^{10}$ J. C. Mitchell and D. J. Meier, J. Polym. Sci., Part A-2 6, 1689 (1968).

${ }^{11}$ M. Yamamoto and J. L. White, J. Polym. Sci., Part A-2 9, 1399 (1971).

${ }^{12}$ D. Luch and G. S. Y. Yeh, J. Macromol. Sci., Phys. 7, 121 (1973).

${ }^{13}$ G. R. Mitchell, Polymer 25, 1562 (1984).

${ }^{14}$ R. N. J. Conradt, B. Heise, and H. G. Kilian, Prog. Colloid Polym. Sci. 87, 85 (1992).

${ }^{15}$ S. Toki, T. Fujimaki, and M. Okuyama, Polymer 41, 5423 (2000).

${ }^{16}$ A. N. Gent and L.-Q. Zhang, J. Polym. Sci., Part B: Polym. Phys. 39, 811 (2001).

${ }^{17}$ S. Toki, T. Z. Sen, D. Valladares, and M. Cakmak, Proceedings of a Meeting of the Rubber Division, Providence, Rhode Island, 24-27 April 2001 (American Chemical Society, Washington, DC, 2001), Paper No. 12.
${ }^{18}$ S. Murakami and A. Kawaguchi, Kobunshi Ronbunshu 54, 183 (1997).

${ }^{19}$ S. Murakami, Y. Nishikawa, M. Tsuji, A. Kawaguchi, S. Kohjiya, and M. Cakmak, Polymer 36, 291 (1995)

${ }^{20}$ S. Murakami, K. Senoo, S. Toki, and S. Kohjiya, Polymer 43, 2117 (2002).

${ }^{21}$ S. Toki, I. Sics, S. Ran, L. Liu, B. S. Hsiao, S. Murakami, K. Senoo, and S. Kohjiya, Macromolecules 35, 6578 (2002)

${ }^{22}$ M. Tosaka, S. Murakami, S. Poompradub, S. Kohjiya, Y. Ikeda, S. Toki, I. Sics, and B. S. Hsiao, Macromolecules 37, 3299 (2004).

${ }^{23}$ M. Tosaka, S. Kohjiya, S. Murakami, S. Poompradub, Y. Ikeda, S. Toki, I. Sics, and B. S. Hsiao, Rubber Chem. Technol. 77, 711 (2004).

${ }^{24}$ M. L. Studebaker, Reinforcement of Elastomers (Interscience Publishers, New York, 1965), p. 319.

${ }^{25}$ S. Poompradub, M. Tosaka, S. Kohjiya, Y. Ikeda, S. Toki, I. Sics, and B. S. Hsiao, Chem. Lett. 33, 220 (2004).

${ }^{26}$ L. R. G. Treloar, The Physics of Rubber Elasticity, 3rd ed. (Clarendon, Oxford, 1975).

${ }^{27}$ S. Toki, I. Sics, S. Ran, L. Liu, and B. S. Hsiao, Polymer 44, 6003 (2003).

${ }^{28}$ S. Toki and B. S. Hsiao, Macromolecules 36, 5915 (2003).

${ }^{29}$ S. Toki, I. Sics, B. S. Hsiao, S. Murakami, M. Tosaka, S. Poompradub, S. Kohjiya, and Y. Ikeda, J. Polym. Sci., Part B: Polym. Phys. 42, 956 (2004).

${ }^{30}$ S. Toki et al., Rubber Chem. Technol. 77, 317 (2004).

${ }^{31} \mathrm{H}$. Westlinning and H. Fleischhauer, Reinforcement of Elastomers (Interscience Publishers, New York, 1965), p. 425.

${ }^{32}$ R. L. Sass, R. Vidale, and J. Donohue, Acta Crystallogr. 10, 567 (1957).

${ }^{33}$ H. P. Klug and L. E. Alexander, X-ray Diffraction Procedures for Polycrystalline and Amorphous Materials, 2nd ed. (Wiley-Interscience, New York, 1974).

${ }^{34}$ S. C. Nyburg, Acta Crystallogr. 7, 385 (1954).

${ }^{35}$ J. Billmeyer and W. Fred, Textbook of Polymer Science, 3rd ed. (WileyInterscience, New York, 1984).

${ }^{36}$ K. Armistead, G. Goldbeck-Wood, and A. Keller, Adv. Polym. Sci. 100, 221 (1992).

${ }^{37}$ D. Luch and G. S. Y. Yeh, J. Appl. Phys. 43, 4326 (1972).

${ }^{38}$ T. Shimizu, M. Tsuji, and S. Kohjiya, Mater. Sci. Res. Int. 4, 117 (1998).

${ }^{39}$ M. Tsuji, T. Shimizu, and S. Kohjiya, Polym. J. (Tokyo, Jpn.) 31, 784 (1999).

${ }^{40}$ M. Tsuji, T. Shimizu, and S. Kohjiya, Polym. J. (Tokyo, Jpn.) 32, 505 (2000).

${ }^{41}$ T. Shimizu, M. Tosaka, M. Tsuji, and S. Kohjiya, Rubber Chem. Technol. 73, 926 (2000).

${ }^{42}$ Y. Miyamoto, H. Yamao, and K. Sekimoto, Macromolecules 36, 6462 (2003).

${ }^{43}$ S. Trabelsi, P.-A. Albouy, and J. Rault, Macromolecules 36, 7624 (2003).

${ }^{44}$ N. Bekkedahl and L. A. Wood, Ind. Eng. Chem. 33, 381 (1941).

${ }^{45}$ W. R. Krigbaum and R.-J. Roe, J. Polym. Sci. A 2, 4391 (1964)

${ }^{46}$ J. Dietrich, R. Ortmann, and R. Bonart, Colloid Polym. Sci. 266, 299 (1988).

${ }^{47}$ S. D. Gehman and J. E. Field, Rubber Chem. Technol. 14, 85 (1941).

${ }^{48}$ L. Bokobza, Macromol. Symp. 169, 243 (2001).

${ }^{49}$ K. Fujimoto, Nippon Gomu Kyokaishi 37, 602 (1964).

${ }^{50}$ S. Fujiwara and K. Fujimoto, Rubber Chem. Technol. 44, 1273 (1971).

${ }^{51}$ E. M. Dannenberg, Rubber Chem. Technol. 59, 512 (1986).

${ }^{52}$ G. Kraus, Rubber Chem. Technol. 51, 297 (1978).

${ }^{53}$ A. I. Medalia and G. Kraus, Science and Technology of Rubber, 2nd ed. (Academic, San Diego, 1994), p. 387.

${ }^{54}$ A. I. Medalia, Rubber Chem. Technol. 46, 877 (1973).

${ }^{55}$ Y. Takahashi and T. Kumano, Macromolecules 37, 4860 (2004).

${ }^{56}$ W. F. Reichert, D. Göritz, and E. J. Duschl, Polymer 34, 1216 (1993).

${ }^{57}$ D. Göritz, Angew. Makromol. Chem. 202/203, 309 (1992).

${ }^{58}$ W. F. Reichert and D. Göritz, Rubber Chem. Technol. 66, 1 (1993).

${ }^{59}$ M. Tsuji, Comprehensive Polymer Science, (Pergamon, Oxford, 1989), Vol. 1, p. 785.

${ }^{60}$ Y. Fukahori and W. Seki, J. Mater. Sci. 28, 4471 (1993).

${ }^{61}$ A. N. Gent, S. Kawahara, and J. Zhao, Rubber Chem. Technol. 71, 668 (1998). 